\title{
Investigación
}

\section{El derecho y su praxis en el deporte, un contexto de reflexión}

\author{
Law and its practice in sport, a context \\ of reflection
}

\section{Pedro Alfonso Castro Campos ${ }^{\star}$}

- Recepción: 22/06/2020 • Aprobación: 16/07/2020 • Publicación: 02/10/2020

Para citar este artículo

Castro Campos, P. A. (2020). El derecho y su praxis en el deporte,

un contexto de reflexión. Dos mil tres mil, 22, e242.

https://doi.org/10.35707/dostresmil/22242

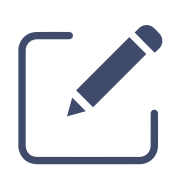

\section{(c) $(1)(9)$}

"Grupo de Investigación Ocio, Cultura Física y Salud-ocufys, Universidad del Tolima, Colombia. orcid: 0000-0002-0428-6670. Correo electrónico: castrocpa@ut.edu.co 
Resumen. El presente artículo tiene como objeto abordar algunas premisas teóricas sobre el derecho y su aplicación en el ámbito deportivo. Se fundamenta en la revisión de artículos y otros referentes bibliográficos. A través del análisis sistemático, como estrategia epistemológica, se genera un marco de reflexión desde el concepto de deporte, su perspectiva competitiva, su trascendencia como fenómeno social, su impacto en el desarrollo económico de Colombia y algunos cambios en la legislación. Se realiza una aproximación holística al derecho del deporte como disciplina, desde varios enfoques: la necesidad de mejorar su aplicabilidad, algunos vacíos, limitantes en su praxis y su potencial. Se reflexiona en torno al tribunal de arbitraje internacional, como mecanismo alternativo en la resolución de conflictos jurídicos deportivos. Por último, se plantean algunas conclusiones producto del análisis.

Abstract. This article aims to address some theoretical premises on law and its application in the sports field. It is based on the review of articles and other bibliographic references. Through systematic analysis, as an epistemological strategy, a framework for reflection is generated from the concept of sport, its competitive perspective, its significance as a social phenomenon, its impact on the economic development of Colombia and some changes in legislation. A holistic approach is made to sports law as a discipline, from various approaches: the need to improve its applicability, some gaps, limitations in its praxis and its potential. It reflects on the international arbitration court as an alternative mechanism in the resolution of sports legal disputes. Finally, some conclusions emerge from the analysis.

Palabras claves

Derecho, deporte, arbitraje jurídico, tribunal internacional.

Key words

Law, sport, legal arbitration, international court. 


\section{Introducción}

No cabe duda de que el deporte es considerado en términos generales una actividad de masas y el impacto que genera debe ser entendido como un proceso social que abarca muchos contextos (Quiñones et al., 2016). “Con gran frecuencia se expresa que el deporte es el fenómeno de carácter cultural más relevante de la sociedad contemporánea” (Quiroga, 2000, s. p.). De esta manera, se evidencia su capacidad de romper barreras socioeconómicas y culturales, tanto así que su estudio compete a diferentes disciplinas: la sociología, psicología, educación, la salud y por supuesto no es ajeno al ámbito del derecho, sobre todo este último, el cual se configura como objeto de análisis en el presente artículo.

Es pertinente señalar que cada día cobran mayor relevancia los análisis que se acercan al contexto del fenómeno deportivo desde lo sociológico y económico, lo cual exhorta el interés académico en los problemas del derecho del deporte, y se afianza en algunos sectores en el estudio de temas jurídicos, que validan la relación entre el deporte y el derecho (Pérez, 2017). Desde esa premisa, el artículo pretende aportar algunos argumentos que sirvan de marco de reflexión y avance de un ejercicio de investigación más amplio.

\section{Aproximación conceptual al deporte}

Son diversas las aproximaciones teóricas al término deporte, algunas más pragmáticas al considerar su polisemia semántica (Diazgranados \& Garzón, 2016). Para ofrecer una perspectiva consistente, se pone a consideración la idea de García (1990) respecto del deporte, que lo define como: "Una actividad física e intelectual, humana, de naturaleza competitiva y gobernada por reglas institucionalizadas" (p. 31). En este sentido, el deporte se configura como actividad competitiva, reglada y de carácter institucional. Por su parte, la definición de Castejón agrega una nueva concepción del deporte, que recoge más características que aforan a cualquier disciplina deportiva:

Actividad física donde la persona elabora y manifiesta un conjunto de movimientos o un control voluntario de los movimientos, aprovechando sus características individuales y/o en cooperación con otro/s, de manera que pueda competir consigo mismo, con el medio o contra otro/s tratando de superar sus propios límites, asumiendo que existen unas normas que deben respetarse en todo momento y que también, en determinadas circunstancias, puede valerse de algún tipo de material para practicarlo. (Castejón, 2001, p. 17).

Por otra parte, desde el ordenamiento legal internacional, se ha buscado ofrecer elementos jurídicos a la actividad deportiva, principalmente las que tienen sentido competitivo, como advierten Diazgranados y Garzón, quienes desde esta noción referencian la Carta Europea del Deporte, en la cual se plantea:

Todo tipo de actividades físicas que, mediante una participación, organizada o de otro tipo, tengan por finalidad la expresión o la mejora de la condición física y psíquica, el desarrollo de las relaciones 
sociales o el logro de resultados en competiciones de todos los niveles. (Diazgranados \& Garzón, 2016, p. 15).

En este concepto se evidencia la caracterización del deporte como actividad física, independiente de su concepción de actividad competitiva, lo cual configura un factor de referencia para el ámbito educativo y cultural, incluido por supuesto el componente personal e individual.

El contexto colombiano cuenta con una definición bastante aceptada en el ámbito jurídico, la Ley 181 de 1995, Ley del Deporte, en su artículo 15 menciona: "Es la específica conducta humana caracterizada por una actitud lúdica y de afán competitivo de comprobación o desafío expresada mediante el ejercicio corporal y mental, dentro de disciplinas y normas preestablecidas orientadas a generar valores morales, cívicos y sociales" (p. 4). Desde esa misma orientación se encuentra la clasificación del deporte: deporte asociado, deporte competitivo, deporte formativo, deporte social comunitario, deporte universitario, deporte aficionado y deporte profesional.

Por otra parte, la trasformación que ha tenido el deporte es de gran proporción en el país, alcanzando un alto nivel de trascendencia mundial, pasó de ser una actividad focalizada y orientada a las necesidades del ocio ${ }^{1}$, a desempeñar un papel fundamental como motor de desarrollo en Colombia, producto de su intervención en la oferta y demanda, que amplían diferentes variables: consumo, inversión, ingresos, empleo, entre otras. Pero no solo en la esfera del deporte de elite o profesional, también en el deporte aficionado o deporte para todos. Sumado a estos aspectos positivos, su incidencia permea otros sectores económicos: el turismo, la construcción, el sector textil, el transporte, generando además otros beneficios inmateriales, los relacionados con el sentir nacional, los sentimientos asociados a los logros deportivos y la buena imagen de Colombia en las competencias internacionales (Ruiz, Muñoz \& Mesa, 2010).

En ese sentido, el deporte tiene una fuerte relación con el contexto que lo rodea. De ahí que en él se vivencian las contradicciones de ese escenario: la espectacularización, fenómenos como: el dopaje, el racismo y la violencia. De este modo, el deporte es inherente a todos los aspectos de la realidad, estéticos: es observable; la técnica: se aprende; es comercial: es un producto que se vende y adquiere el criterio de negocio; político: exalta lugares, separa fronteras; medicina: requiere aspectos físicos y corporales (Aceti et al., 2015).

Cabe precisar que el deporte tiene una serie de referentes normativos, los mismos que han permitido sentar las bases de una cultura del deporte, no solo desde la óptica del deporte de elite o la denotación de deporte competitivo, también desde un enfoque de inclusión o deporte para todos.

De acuerdo a los referentes normativos y teóricos, Colombia ha experimentado una serie de cambios en el plano del deporte (Tabla 1), desde la aparición de Comité Olímpico Colombiano (COC) en 1936, hasta la reciente creación del Ministerio del Deporte en 2019, este paso entendido como uno de los mayores logros del ámbito normativo del deporte colombiano, al

${ }^{1}$ Ocio: entendido como vivencia humana, en la cual la persona es protagonista y que puede suponer una experiencia de desarrollo formativo. 
marcar un hito de evolución muy acorde con las políticas de construcción de paz y desarrollo social, al reconocer el deporte como herramienta fundamental, al respecto: "El objetivo de la política pública del deporte en Colombia se orienta a fines superiores en el marco de la estrategia de desarrollo social del país, hacia el desarrollo humano, la convivencia y la paz" (Ortegón Yáñez, 2013, p. 87).

Tabla 1. Algunos antecedentes de la legislación del deporte en Colombia

\begin{tabular}{|c|l|}
\hline Año & \multicolumn{1}{|c|}{ Descripción } \\
\hline 1936 & Se crea el Comité Olímpico Colombiano (coc). \\
\hline 1968 & $\begin{array}{l}\text { Se crea Coldeportes Nacional, el Sistema Nacional del Deporte, la educación } \\
\text { física y la recreación. }\end{array}$ \\
\hline 1970 & Decreto 1387: reglamenta la organización deportiva del país. \\
\hline 1984 & Decreto 3115: se crea la Escuela Nacional del Deporte. \\
\hline 1984 & Decreto 2845: organización del deporte, la educación física y la recreación. \\
\hline 1984 & Decreto 3158: normas clubes profesionales. \\
\hline 1985 & Decreto 1421: reglamenta el Decreto 2845 de 1984 Código disciplinario. \\
\hline 1993 & Ley 49: establece el régimen disciplinario del deporte. \\
\hline 1995 & Ley 181: Ley del Deporte. \\
\hline 1995 & Decreto 1230: reestructura Coldeportes. \\
\hline 1996 & $\begin{array}{l}\text { Decreto 407: reglamenta el reconocimiento deportivo, desde el Sistema Na- } \\
\text { cional del Deporte. }\end{array}$ \\
\hline 1996 & Decreto 776: normas clubes deportivos profesionales. \\
\hline 2001 & Decreto 641: se crea el Comité Paralímpico Colombiano. \\
\hline 2003 & Decreto 1746: adscribe a Coldeportes al Ministerio de Cultura. \\
\hline 2009 & Decreto 1267: Estatuto del Aficionado al Fútbol en Colombia. \\
\hline 2011 & Ley 1445: modifica la Ley 181 de 1995 (cambios a nivel administrativo e \\
institucional al interior del deporte nacional).
\end{tabular}

Fuente: Ministerio del Deporte (s. f.), Oliveros (2019) y Contecha (1999) 


\section{El derecho aplicado al deporte}

El derecho del deporte se viene consolidando en el plano internacional como una disciplina consagrada al estudio de temas jurídicos que se despliegan en el deporte, en ese sentido: "Es la unión entre las diferentes ramas del derecho, que tienen que ver con las distintitas modalidades deportivas" (Silva Londoño, 2014, p. 9). Al respecto, Gil, menciona:

El derecho del deporte es la disciplina que se encarga de abordar el fenómeno deportivo desde las distintas vertientes del derecho, y a la vez posibilita generar intercambios interdisciplinarios que permiten analizar con mayor amplitud y riqueza científica todas las manifestaciones del objeto de estudio: el deporte. El derecho del deporte enfoca los distintos aspectos que presenta el deporte en sus diversas manifestaciones, pero cuidando y respetando la esencia de dicha actividad. Seguido a esto se suma la importancia de los aportes que hacen disciplinas tales como la economía del deporte, la sociología del deporte, la antropología del deporte, la psicología del deporte, la medicina del deporte, etc. (Gil, 2001, p. 34).

La disciplina se ha orientado al estudio de normas emergentes y de un gran número de sistemas de resolución de conflictos, que aparecen en el panorama del deporte, algunos argumentan que el interés de analizar el ámbito deportivo, muchas veces desde la óptica jurídica, se implementa de forma independiente, de ese modo varios países han optado por incorporar diferentes regímenes transnacionales dedicados a estas disciplinas jurídicas al servicio y funcionamiento del deporte, lo cual ha generado en algunos contextos mayor eficiencia que en otros (Avendaño, s. f.).

Desde esa perspectiva: "La circunstancia de que el derecho del deporte toma normas y principios de otras ramas del derecho no puede descalificar la autonomía de esta nueva disciplina normativa, por el contrario, reafirma su independencia" (Clerc, 2012, p. 31). Es necesario tener en cuenta que generalmente ninguna de las ramas tradicionales en las cuales se divide el derecho, brindan una respuesta consistente a los diversos conflictos, dada su alta complejidad y variaciones que suelen aparecer en el ámbito deportivo. Colombia refleja un nivel de poca eficiencia del sistema jurídico nacional para la resolución de casos en materia de deporte, que exhortan a la reflexión sobre las falencias en este ámbito, además de considerar que existe sustento en normas nacionales que enmarcan el deporte y la jurisprudencia del tema (Avendaño, s. f.).

Otro atenuante, es la dinámica de la industria deportiva en Colombia, la cual presenta una tasa de crecimiento significativa, configurando un escenario que empieza a aprovechar paulatinamente su potencial ligado con el derecho, cifras estadísticas ratifican que es un sector de reconocido impacto económico: "El deporte es un sector económico que ha ganado importancia en el mundo, debido a la gran acogida de sus espectáculos en la sociedad. A raíz de esto muchos se han preguntado qué tanto es el impacto económico" (Ruiz, Muñoz \& Mesa, 2010, p. 165). $\mathrm{Al}$ establecer la necesidad de contar con medios para verificar ese crecimiento económico en los clubes y otros sectores relacionados con el deporte, algunas particularidades propias de las relaciones entre entidades deportivas, temas contractuales de los deportistas, se constituyen 
escenarios que reclaman abogados con conocimiento específico sobre legislación y derecho deportivo.

En ese orden, el apogeo del deporte como fenómeno se ha extendido hacia el derecho; a la luz de esa dinámica, el sector responde con normas cada vez más vigentes, mejor estructuradas, las cuales deben ser interpretadas y aplicadas por los abogados, en ese sentido:

Se configura como una rama jurídica catalizadora de expectativas y experiencias socio-políticoeducacionales-económicas en el plano deportivo compatibilizándolas con un ius singulare ${ }^{2}$ que condensa normas de organización, normas de conducta, normas sustantivas y normas procesales. (Clerc, 2012, p. 21).

Otra perspectiva que vale precisar, radica en la inconformidad de algunos sectores al afirmar que el deporte goza de ciertos privilegios y hasta de un cierto grado de impunidad a la hora de juzgar determinadas conductas desde lo disciplinario, reclamando la necesidad de revisar ese código deportivo, analizar el tipo de faltas, y los criterios bajo los cuales pueden o no trascender al plano penal o civil:

Con base en lo anterior, se podría cuestionar, sí, la conducta antideportiva que infringe el reglamento y provoca lesiones deportivas graves o hasta la muerte, que cuestione al ser juzgada al amparo de la ley disciplinaria deportiva: ¿Cuál debería ser su alcance en materia de responsabilidad penal y civil? (Blanco, 2019, p. 196).

En ese sentido, es importante reflexionar, seguir un estudio crítico y objetivo del marco de responsabilidades en el deporte, considerar que no acatar las normas o reglas dispuestas por cada federación en muchos casos puede comprometer la integridad del propio deportista o de su rival, aparecen otras conductas antideportivas: el dopaje, amaño de resultados y el comportamiento violento, entre otras. Son ejemplos para retratar algunos riesgos, que en determinadas circunstancias pueden trascender el tema disciplinario. Hace varios años, Valls (2009) cuestiona los aspectos que eximen al deporte de las responsabilidades: "La competición deportiva, entendida como un espectáculo, es un valor de las sociedades actuales, pero de ahí a considerar que tiene que ser protegido por el derecho penal no me parece lo acertado" (p. 24). En esa misma línea argumental, Valls complementa lo siguiente:

Cuando hablamos de actividad deportiva nos encontramos con que se trata de una actividad social que se rige por un sistema propio de reglas, que casi siempre difieren en cada deporte. Es justamente el no respetar este conjunto propio de normas escritas y consensuadas lo que se considera una conducta antideportiva. Todos los participantes de tal especialidad conocen y aceptan tomar parte en la misma porque poseen una visión anterior del comportamiento que implica. (Valls, 2009, p. 24).

\footnotetext{
${ }^{2}$ Ius singulare: es una expresión latina. Se traduce literalmente como ley singular. Era una ley especial para ciertos grupos de personas, cosas o relaciones jurídicas (debido a que es una excepción a los principios generales del ordenamiento jurídico).
} 
Dadas estas circunstancias que rodean el derecho aplicado al deporte, las cuales se han expresado líneas atrás y muchos otros enfoques de análisis que no se focalizan en este artículo, surgen muchos interrogantes y posibles líneas de acción. En este punto, para evitar algunas ambigüedades teóricas y respetando el enfoque del artículo, se pone a consideración lo siguiente: "[...] El derecho deportivo es aquel que define los lineamentos para la práctica deportiva y el derecho del deporte es la unión de conceptos que sirven para orientar las relaciones derivadas de las actividades relacionadas con la práctica del deporte" (Silva Londoño, 2014, p. 10).

Aunque el deporte es un campo que identifica la unidad nacional de muchos territorios y es eje de seguimiento por un gran volumen de personas, se evidencia que poco se ha indagado en Colombia sobre su relegación jurídica (Diazgranados \& Garzón, 2016). Desde esta visión, para consolidar un verdadero ordenamiento jurídico y disciplinas que den respuesta pertinente en este campo, se requieren procesos de investigación sistemáticos, fundamentados en: análisis del papel del deportista, actividades que ejerce, las federaciones y otras variables objeto de estudio en el marco de la profesión.

No obstante, como panorama emergente y paralelo a estas necesidades, existen otros escenarios sobre todo en el contexto internacional, espacios que paulatinamente se ocupan de resolver algunos problemas del ámbito legal del deporte, integrados por diversas normas jurídicas de diferente origen, nacionales y extranjeras, de naturaleza pública y privada, cuentan con la intervención de instituciones deportivas de varios países y culturas jurídicas, producto de la globalización deportiva, que aumentan en gran medida el nivel de las controversias, gestan un marcado posicionamiento emergente, reclaman el espacio de normas y leyes propias del deporte, y suscitan un panorama de amplia jurisprudencia sobre todo en los llamados tribunales de arbitraje (Ortega, 2014).

\section{Mecanismo alternativo: Tribunal Arbitral du Sport (TAS)}

Es necesario señalar que un alto porcentaje de los conflictos del ámbito deportivo, se desarrollan bajo la intervención de mecanismos alternativos en la resolución de conflictos en el deporte, organismos como el Tribunal Arbitral du Sport (TAS) ${ }^{3}$ (Giraldo, 2017). Esto, al considerar muchos factores, principalmente la falta de instituciones u organismos independientes que desarrollen esa labor en el contexto internacional, específicamente en el escenario latinoamericano, aunado a la influencia que tienen gran parte de las federaciones deportivas sobre sus asociaciones en direccionar al TAS como espacio de apelación, en ese sentido se pueden referenciar algunas ventajas (Echeverría, 2017).

La fluidez es un aspecto fundamental, principalmente su procedimiento con menos burocracia en corporación a la justicia ordinaria; este componente tiene una gran connotación de rapidez en el referente jurídico deportivo y sin duda en el mundo del deporte, la solución ágil

\footnotetext{
${ }^{3}$ (TAS), por sus siglas en francés (Tribunal Arbitral du Sport), es una entidad jurisdiccional que soluciona los asuntos más importantes en torno al deporte, con facultades de un órgano común.
} 
de un conflicto adquiere un plus de relevancia mayor, al entender las dinámicas de competencia, calendarios de los torneos y los periodos de alto rendimiento del deportista, que en muchos casos se ven limitados esperando sentencias judiciales (Ortega, 2014). No obstante, razones de costos y el idioma en los arbitrajes jurídicos, configuran una gran dificultad para los latinoamericanos a la hora de llegar a esta instancia (Giraldo, 2017). A pesar de la corta duración de los procesos y también que dichos costos se establecen con antelación.

Otro rasgo importante es la posibilidad de las partes de someterse a la decisión de un tercero, que tiene gran experiencia y conocimiento en campo. Es una institución imparcial e independiente, la posibilidad de buscar mediadores internacionales, árbitros imparciales que no estén relacionados con las federaciones y por tal razón no tengan ninguna influencia en las decisiones (Universidad Externado de Colombia, 2019). La privacidad se maneja con gran equilibrio, manteniendo los niveles adecuados de confidencialidad, sobre todo alejado de efectos políticos nocivos, pero con un margen de información coherente con la sociedad (Ortega, 2014).

Es adecuado mencionar la clasificación en el arbitraje según la materia: arbitraje en derecho, en equidad y técnico. El primero en mención exige que los árbitros sean abogados titulados, teniendo en cuenta que deciden con base en normas jurídicas. En la segunda modalidad, los árbitros deciden en función del sentido común y razones de equidad, de este modo no exige que sean abogados. El arbitraje técnico sí requiere de conocimientos propios de la disciplina deportiva, un nivel profesional, especializados, dependiendo del campo en el cual sea el litigio (Perico, Beltrán \& Gómez, 2012).

Los conflictos en materia deportiva pueden dividirse en dos clases: contractuales y disciplinarios, es muy frecuente encontrar controversias de naturaleza disciplinaria, producto de comportamientos antideportivos, en la mayoría de circunstancias por conductas contrarias a los deberes que demanda su rol profesional, apareciendo el dopaje como la conducta más reprochable socialmente (Perico et al., 2012). Cabe mencionar que las decisiones emitidas por el TAS son de carácter resolutivo, que equivale a una sentencia en un proceso jurisprudencial ordinario. De este modo, la sentencia declara el final de la discusión que se pretende resolver (Universidad Externado de Colombia, 2019).

Por último, en el contexto del deporte colombiano, específicamente en el Código Disciplinario Único de la Federación Colombiana de Fútbol (FCF), al respecto del TAs, señala:

Artículo 3. Obligación de sometimiento de controversias ante los órganos deportivos y a los tribunales de arbitramentos fijados en asamblea. De conformidad con los Estatutos de la Fifa y la сопмевоL, las Divisiones, las ligas, los clubes aficionados y profesionales, oficiales, jugadores y cuerpo técnico que hacen parte de la organización del fútbol colombiano o cualquier otra persona vinculada a FCF o sus divisiones de manera directa o indirecta, están en la obligación de someter sus diferencias y toda controversia de orden disciplinario y relacionada con materias de libre 
disposición en términos legales a la decisión de los órganos disciplinarios deportivos y acatar y cumplir sus decisiones.

En virtud de lo anterior, no podrán someter disputas de tal naturaleza ante tribunales ordinarios a menos que se especifique en la reglamentación Fifa. Agotada la vía federativa (instancias de los órganos disciplinarios deportivos) podrán someter las controversias ante el Tribunal Arbitral de Deporte (TAS) de Lausana, Suiza, o al tribunal de arbitramento que eventualmente se señale en los estatutos de la FIFA, la CONMEBOL, la FCF y la DiMAYOR según corresponda. (FCF, 2019, pp. 2-3).

\section{Conclusiones}

En Colombia ha sido loable el interés desde el ámbito normativo por regular el deporte; pese a esto, se requiere una mayor precisión en el tema, más congruencia con los estándares internacionales, labrar un camino que permita de forma paulatina hablar realmente de una disciplina del derecho, autónoma e independiente.

En ese sentido, los problemas frutos de este vacío normativo justifican la necesidad de constituir un derecho deportivo autónomo e independiente en Colombia, que regule las relaciones derivadas del deporte. Se debe considerar que el derecho del deporte viene incorporando a su ejercicio normas y principios de otras ramas del derecho, pero esa categoría no le hace perder su estatus autónomo, en la medida que ninguna de las ramas tradicionales del derecho tiene los elementos suficientes para dar respuestas precisas a muchos de los conflictos que nacen de las relaciones jurídicas específicas del campo del deporte, que generen la necesidad de establecer un cuerpo normativo capaz de garantizar soluciones pertinentes e integrales.

Pese a lo anterior, el interés de configurar al deporte como una rama autónoma susceptible de regulación particular desde el derecho, no ha crecido en la misma proporción en los diferentes países, algunos cuentan con organismos e instrumentos que se encargan de regular las diferentes disciplinas deportivas. En el contexto colombiano el avance en esta materia ha sido lento y de menor impacto en la consolidación de la industria deportiva.

En Colombia, el deporte como factor de desarrollo económico demanda personas que desplieguen y apoyen estas disciplinas de manera profesional, al considerar el desarrollo actual, la gran envergadura de recursos económicos y humanos que concibe, lo cual genera la necesidad de abogados con prácticas y conocimientos concretos del derecho orientado al deporte.

Desde esa óptica, el derecho aplicado al deporte configura una asignatura muy compleja, la cual está en constante evolución, que además reclama conocimientos interdisciplinarios entre las diferentes áreas del derecho: derecho laboral, la propiedad intelectual, aspectos tributarios y financieros, entre otros. En ese orden, ahora las empresas deportivas son efectivas organizaciones comerciales y los deportistas se configuran como marca personal, haciendo necesaria la formación en este espacio del derecho, como respuesta a su crecimiento exponencial y dinámica de cambio. Por ende, gestionar un centro de arbitraje deportivo en Colombia, cala perfectamente en el marco de los lineamientos establecidos en la Ley 1563 del 2012, se configura como 
una opción pertinente, para responder a las necesidades del contexto local y superar el tema de costos e idiomas.

Para finalizar, es imperativo señalar que el derecho internacional no ha permanecido al margen de la regulación de cuestiones de índole deportiva. Incluso la cooperación internacional ha gestado resultados trascendentales en la lucha contra el dopaje y el amaño de resultados deportivos. "El deporte tiene el poder de cambiar el mundo. Tiene el poder de ilusionar, tiene el poder de unir a la gente de una manera que pocas cosas lo pueden hacer" (Mandela, citado por Tejero, 2016, p. 6).

\section{Referencias}

Aceti, E., Castelli, L., López, C., Mogni, J., Lamoneda, J., Ziegler, J., \& Heid, S. (2015). Desarrollo social a través del deporte. Quito, Ecuador: Universidad Andina Simón Bolívar, Sede Ecuador. Recuperado de http://scioteca.caf.com/handle/123456789/734

Avendaño, Herrera, J. (s.f.). Informe de investigación sobre el marco jurídico (legal y jurisprudencial) del derecho deportivo: Análisis de problemáticas en los sistemas de resolución de conflictos (Tesis de derecho). Facultad de Derecho Universidad de los Andes, Bogotá, Colombia. Recuperado de http://hdl.handle.net/1992/18542

Blanco Zúñiga, O. (2019). La responsabilidad penal y disciplinaria en el derecho deportivo en Colombia. Revista digital: Actividad física y deporte, 6 (1), 194-204 Recuperado de https://revistas.udca.edu.co/ index.php/rdafd/article/view/1440/

Castejón, F, J. (2001). Iniciación deportiva. Aprendizaje y enseñanza. Madrid, España: Pila Teleña.

Clerc, C. (2012). Derecho del Deporte o Derecho Deportivo. Revista de Derecho, Escuela de Postgrado, (2), 17-34. Recuperado de https://revistas.uchile.cl/index.php/RDEP/article/view/31001

Colombia. Ley 181 de 1995. Ley del Deporte. Por el cual se dictan disposiciones para el fomento del deporte, la recreación, el aprovechamiento del tiempo libre y la Educación Física y se crea el Sistema Nacional del Deporte. Recuperado de https://inst-mpal-recreacion-y-el-deporte-de-soacha.micolombiadigital.gov.co/sites/inst-mpal-recreacion-y-el-deporte-de-soacha/content/files/000146/7282_ ley_181_de_1995.pdf

Contecha Carrillo, L. F. (1999). La Educación Física y el Deporte en Colombia. Una historia. Lecturas: Educación Física y Deporte, (17). Recuperado de https://www.efdeportes.com/efd17/efcolom.htm

Diazgranados Quimbaya, C \& Garzón Landínez., T. (2016). Régimen laboral del derecho deportivo colombiano, Bogotá, Colombia: Universidad Católica de Colombia. Recuperado de https://repository.ucatolica.edu.co/bitstream/10983/14423/4/regimen-laboral-del-derecho-deportivo-colombiano.pdf

Echeverría Bermúdez, M. (2017). La Corte Arbitral de Deportes (TAS-CAs) como mecanismo moderno para la resolución de disputas en el fútbol profesional. Foro Jurídico, (11), 91-98. Recuperado de http://revistas.pucp.edu.pe/index.php/forojuridico/article/view/18578/

Federación Colombiana de Fútbol (2019). Código Disciplinario Único Federación Colombiana de Fútbol-FCF. Recuperado de https://fcf.com.co/index.php/2019/04/22/codigo-disciplinario-unico/ 
García Ferrando, M. (1990). Aspectos sociales del deporte: Una reflexión sociológica. Madrid, España: Alianza.

Gil Domínguez, A. (2001). El derecho al deporte y el derecho del deporte. En Cuadernos de Derecho Deportivo. No 1, 17-34. Buenos Aires, Argentina: Editorial Ad Hoc.

Giraldo, C. (2017, 29 de septiembre). La importancia de implementar un sistema de arbitraje deportivo en Colombia. Legis Ámbito Jurídico. Recuperado de https://www.ambitojuridico.com/noticias/educacion-y-cultura/la-importancia-de-implementar-un-sistema-de-arbitraje-deportivo-en

Ministerio del Deporte de Colombia (s. f.). Inspección Vigilancia y Control. Recuperado de https://www. mindeporte.gov.co/index.php?idcategoria $=37475 \& p a g=4$

Oliveros Gabriel, S. (2019). Registro normativo y desarrollo de la legislación para el deporte nacional. Revista Digital: Actividad Física y Deporte, 5 (2), 197-220. https://doi.org/10.31910/rdafd. v5.n2.2019.1268

Ortega Sánchez, R. (2014). Arbitraje jurídico deportivo. Diálogos de Saberes, (41), 47-66. https://doi.org/10.18041/0124-0021/dialogos.41.199

Ortegón Yáñez, R. (2013). Política pública del deporte en Colombia. En F. Tabares. (Ed.), Educación física, deporte, recreación y actividad física: Construcción de ciudadanías (pp. 86-110). Medellín, Colombia: Funámbulos Editores. Recuperado de http://viref.udea.edu.co/contenido/publicaciones/expo2013/ libro-academic-Expomotricidad2013.pdf

Pérez González, C. (2017). ¿Un derecho internacional del deporte? reflexiones en torno a una rama del derecho internacional público in statu nascendi. Revista Española de Derecho Internacional, 69 (1), 195-217. http://dx.doi.org/10.17103/redi.69.1.2017.1.07

Perico Peña, D., Beltrán, N., \& Gómez Acuña, D. (2012). Análisis del derecho deportivo desde la perspectiva del derecho comercial [Trabajo de grado Especialización Derecho Comercial]. Pontificia Universidad Javeriana. Bogotá, Colombia. Recuperado de http://hdl.handle.net/10554/10003

Quiñones Valero, A., Gordon, D., De la Plata, N., Muñoz, M., Torres, J., \& Peña, J. (2016). Las políticas públicas del deporte, la recreación y la actividad física en Colombia: Un análisis contextual y de referentes exitosos. Bogotá, Colombia: Coldeportes; Universidad Sergio Arboleda. Recuperado de http:// hdl.handle.net/11232/943

Quiroga, S. R. (2000). Democracia, comunicación, cultura popular y deporte. Lecturas: Educación Física $y$ Deporte, (18). Recuperado de https://www.efdeportes.com/efd18a/democ.htm

Ruiz Molina, A, Muñoz, E., \& Mesa, R. (2010, enero-junio). Medición económica del deporte en Colombia: Una propuesta metodológica de cuenta satélite. Lecturas de Economía, (72), 141-167. Recuperado de http://www.scielo.org.co/pdf/le/n72/n72a06.pdf

Silva Londoño, D. (2014). Los efectos y alcances del derecho del deporte y su influencia en la responsabilidad civil colombiana [Trabajo de grado para optar al título de abogado]. Pontificia Universidad Javeriana. Bogotá, Colombia. Recuperado de http://hdl.handle.net/10554/34418

Tejero González, C. (2016). Sobre la importancia del deporte como acción política: Razones y medidas de gobernanza. Cultura, Ciencia y Deporte, 11(31), 5-6. Recuperado de https://www.redalyc.org/ pdf/1630/163044427001.pdf 
Universidad Externado de Colombia (2019). Guía Académica - Tribunal Arbitral del Deporte. Modelo de Naciones Unidas. Bogotá, Colombia. Universidad Externado de Colombia-Subsecretaría de Comités Jurídicos. Recuperado de https://www.uexternado.edu.co/wp-content/uploads/2019/12/ Gu\%C3\%ADa-Acad\%C3\%A9mica-Tribunal-Arbitral-del-Deporte.pdf

Valls Prieto, J. (2009). La intervención del derecho penal en la actividad deportiva. Revista Electrónica de Ciencia Penal y Criminología, (11), 1-25. Recuperado de http://criminet.ugr.es/recpc/11/recpc11-14.pdf

\section{Bibliografía recomendada}

Aristizábal Botero, M. (2013). Hermenéutica del vicio constitucional en la reglamentación del derecho al deporte en Colombia. Revista Pensamiento Jurídico, (36), 61-88. Recuperado de https://revistas. unal.edu.co/index.php/peju/article/view/40317 